\title{
Improved energy efficient hierarchical cluster tree based routing to prolong network lifetime
}

\author{
Venkatesh Prasad B S \\ Research Scholar \\ Department of CSE \\ GSSSIETW, Mysuru , INDIA \\ venkyp25@gmail.com \\ (Author 1)
}

Abstract:

Wireless sensor networks (WSN) play a key role in enabling wireless communication technology among several nodes to remotely communicate and exchange information. WSN consists of tiny sensor nodes equipped with battery, scattered in an area to gather information around an environment and send to data collection node known as sink or base station (BS). WSN have been widely used in various applications like agriculture, fire detection, health care and military and has become imperative necessity for future revolutionary area like UAV (unmanned aerial vehicles), IoT (Internet of things) and smart cities which employs large scale sensor nodes. However sensor nodes are limited to battery, memory, low computational power, resource and bandwidth. Continues sensing of events, makes node to drain its battery faster and goes dead fast. For resource constrained WSN, hierarchical cluster based approaches are considered as energy efficient and improves network performance for large scale WSN. Minimizing energy consumption and extending network lifetime are major challenging issues of WSN, clustering methods with optimized routing have offered solution to optimize energy utilization. To balance energy consumption and improve network lifetime many existing hierarchical clustering approaches have been proposed, however existing method does not consider rotation of cluster head (CH) and considers cluster head selection based on residual energy and distance parameter. In this paper we propose an improved energy efficient cluster tree (IEECT) based routing to improve energy efficiency of hierarchical cluster. IEECT considers modification of existing LEACH (Low energy adaptive clustering hierarchy) protocol to improved energy efficient LEACH by considering energy parameters like residual node energy and

\author{
Roopashree H R \\ Associate Professor \\ Department of CSE \\ GSSSIETW, Mysuru, INDIA \\ roopashreehr@gsss.edu.in \\ (Author 2)
}

average network energy. IEECT accounts optimal number of cluster head $(\mathrm{CH})$ and selection of $\mathrm{CH}$ is done using threshold value among sensor nodes. Proposed IEECT combines tree based routing and data aggregation scheme to maintain desirable quality of service. Simulation experiments are carried out by using network simulator. Performance of IEECT is evaluated in terms of PDR, delay, energy consumption, network lifetime and overhead.

Keywords: Cluster, data aggregation, energy efficient, lifetime, routing, WSN

\section{Introduction}

Wireless sensor network (WSN) is a self-organizing and autonomously distributed system consisting of several tiny sensor nodes scattered in an area to continuously monitor the physical environment to gather information and send it to base station (BS) or sink [1]. Sensor nodes work independently without human intervention and are limited to bandwidth, computation power, memory and energy source [2]. Advancement in low power embedded systems brings light weighted sensors used for numerous real time applications such as smart cities, military, health care, industry automation and agriculture [3-9]. Information gathered by sensor nodes will be routed by cooperating with nodes and creating a routing path to destination. Finally the information is processed and analyzed in timely manner to avoid economic loss [10]. Collected information is transmitted over wireless communication channel [11], sensors depend on battery power for sending and receiving data. Consuming more power results in sensor death and decreases overall network lifetime. In most of the sensor applications replacement of batteries is not practical after begin deployed in harsh environment. Therefore optimizing sensors energy consumption is an important and challenging issue for large scale sensor 
network. Hierarchical cluster based routing methods have advantages related to energy efficiency and scalability [12]. Hierarchical cluster based routing methods have been proven an efficient way to increase overall network lifetime and reduce energy consumption of sensor node within clusters by performing information aggregation [13]. In clustering the network is divided into group of clusters based on set of criteria. Each cluster has leader known as cluster head $(\mathrm{CH})$, elected based on high energy which is responsible to gather information from sensors or cluster members (CM), aggregate and forward it to sink or base station (BS) [14]. Clustering method can balance work load of sensor nodes and minimize the overall energy consumption. Low energy adaptive clustering hierarchy protocol (LEACH) is considered to be most prominent clustering method [15]. Network lifetime in LEACH is divided into rounds which consist of two phase setup and steady phase. However in LEACH the selection of cluster head $(\mathrm{CH})$ is done randomly and considers only energy parameter while selecting $\mathrm{CH}$, which does not guarantee the selection of reliable node to act as cluster head in each round. Furthermore LEACH uses single hop communication between $\mathrm{CH}$ and base station which consumes more energy when cluster head is far from base station making it as disadvantage. In this paper we propose an improved energy efficient cluster tree (IEECT) based routing by modifying existing $\mathrm{LEACH}$, the main contributions are as follows:

- IEECT considers optimal cluster head selection by setting threshold parameter in each round and also considering parameters like nodes initial energy, residual energy, average and total energy which existing LEACH does not considers as important parameters.

- Constructing logical tree among the cluster head to sink reduces the distance between cluster head and sink node for data transfer which consumes less energy and reduces communication overhead. IEECT also combines tree based data aggregation scheme which reduces the traffic load and improves network lifetime.

\section{Problem definition and Motivation}

Wireless sensor network (WSN) gather, analyze and utilize data received from the sensor and have been used for various real time applications. Continuous sensing of event around the sensing area decreases battery of sensor node, which may result in malfunctioning nodes and interrupt service. For large scale WSN, replacement of sensor battery becomes inevitable which is a limiting factor in WSN. Due to energy constrain improvement of network lifetime becomes crucial and routing the data to process at sink with effective battery power is still a challenging issue. In distributed routing each node considers local information and autonomously makes the routing decision rather considering global network information which causes loop formation and collusion. To overcome energy and routing problem, hierarchical cluster based routing concept has been applied to achieve energy efficiency and low communication overhead in WSN. Clustering method optimizes energy consumption of sensor nodes and avoids duplicate data transfer to sink. High energy nodes known as cluster head $(\mathrm{CH})$ plays a crucial role in gathering and aggregating collected data from sensor node or cluster members and forward it to sink. Packet failures occur due to inefficient selection of cluster head and causes faster battery drain of nodes nearer to sink which leads to energy hole problems. However clustering approaches have drawbacks. Existing LEACH does not form optimal cluster heads and does not provide localized information of sensor. LEACH does not calculates optimal distance between cluster head to sink and makes all the cluster head to connect with sink, resulting nodes near to sink consuming high energy. Cluster head is selected randomly and is kept on round the clock making it damaged quicker than other nodes. To extend network lifetime it is essential to construct optimal clusters, rotation of cluster head balances nodes energy, giving chance to other nodes becoming cluster head based on their residual energy. To alleviate routing loop tree based data aggregation routing optimizes data transmission paths and prolongs network lifetime. Therefore this motivates us to design an energy balance scheme to extend network lifetime.

The whole paper is organised as follows: Section I describes Introduction, section II describes problem definition and motivation, section III describes related works done, section IV describes the network model and preliminaries considered in this paper, section $\mathrm{V}$ describes the proposed IEECT scheme, section VI describes the results and performance evaluation and finally conclusion is drawn in section VI

\section{Related Works}

In [16] author proposed LEACH protocol aiming to reduce energy consumption of nodes by employing random number generation by each node. The result of random number is compared with parameter that 
represents the chance of node becoming cluster head. At different rounds, the generated value increases if the node is not elected as cluster head and it is determined by parameter function $p$. Node becomes cluster head if the random number is greater than $p$. In [17] author proposed an adaptation method using LEACH where different thresholds are set by nodes depending on residual energy and distance to sink. However in LEACH protocol cluster head is always kept on, cluster head consumes huge amount of energy to transfer data to sink through single hop. If any cluster head is damaged, cluster will have no use. In [18] author aims to solve load balancing problem in clustering method by proposing fixed parameter tractable (FPT) algorithm. FPT consists of four phase, node information like location and their neighbours are collected and sent to sink in first phase. Clusters are formed based on different criteria in second phase. Gateway nodes are determined to link sink node through cluster head for transmitting data through multihop in third phase. Data aggregation and re-clustering is done in fourth phase. FPT algorithm has increased network lifetime and throughput, drawback of this method has higher overhead and consumes more energy at different rounds. In [19] author proposed fuzzy based cluster routing with constant threshold to enhance network lifetime. In this routing algorithm periodic formation of cluster is avoided by fixing threshold value and multihop routing is utilized for sending data to base station (BS). $\mathrm{CH}$ are selected by using fuzzy logic based on nodes residual energy. In multihop routing next hop $\mathrm{CH}$ nodes are selected based on the distance to base station and remaining energy. This routing method reduces the control overhead messages and enhances network lifetime, however this method fails to provide network stability and increases delay. In [20] author proposed cluster based efficient energy aware routing protocol to reduce nodes energy consumption. Protocol composed of layers of clusters till sink node. Network lifetime is similar to $\mathrm{LEACH}$, consisting of round time specified by sink. This routing scheme consist of dual cluster head namely $\mathrm{CH}$ and leader head, leader head is used to collect and aggregate data from sensor node and $\mathrm{CH}$ is used to relay data between layers until it reaches to sink. Leader head is selected based on its location and energy, while $\mathrm{CH}$ is chosen based on energy and distance to sink. Major drawback of this routing scheme is that it generates more number of control messages during multihop routing causing higher communication overhead and decrease network lifetime. In [21] author proposed improved artificial bee colony algorithm for optimal clustering and scheduling. Fitness function was used to calculate metrics like residual energy, transmission power and distance to sink from cluster head. Optimal cluster head selection is done using optimization algorithm, however this algorithm lacks to collect and analyze secondary information of cluster members which results in frequent formation of cluster head. Summary of the above discussed related works are related to energy optimization and efficient cluster head selection to improve network lifetime. Authors considered parameters like single hop cluster communication and cluster head selection based on residual energy, however to balance nodes energy and to avoid early node death parameters like initial energy, total network energy and residual energy parameters has to be considered. In this work we consider rotation of cluster head by considering parameter including initial energy, total network energy, residual energy to form optimal cluster head and balance nodes energy further features of tree based data aggregation is used to route aggregated data reliably to sink.

\section{Network Model and Preliminaries}

Network model consist of sensor nodes organized in hierarchical design, which has better performance than flat network in terms of communication overhead, energy consumption [22]. Network is divided into groups of cluster, each cluster consists of cluster head (CH) which gathers data from sensor or cluster members $(\mathrm{CM})$ and aggregates collected data within cluster and forward it to sink or base station (BS). Figure 1 shows the network architecture.

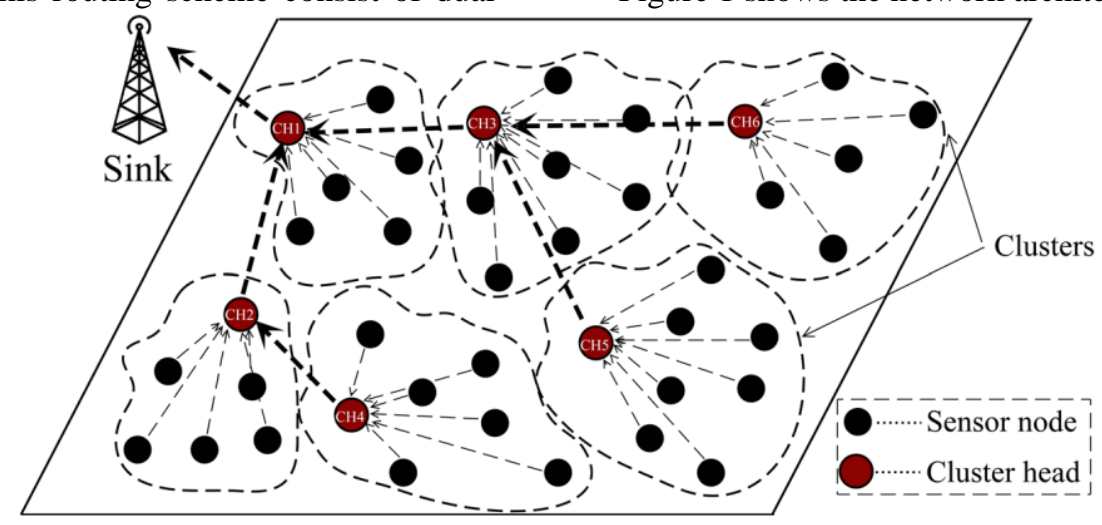




\section{Figure 1: Network model of IEECT}

\section{Preliminaries}

- Network consists of sensor nodes deployed uniformly in a sensing area and are homogenous and assigned with same initial energy [23, 24].

- Sensor nodes and sink node is assumed to be stationary after their deployment, sink is placed relatively far from WSN and has no energy constrain.

- Nodes can readjust their transmission power depending on the situation.

- When nodes energy is completely exhausted, it is termed as dead node since nodes battery cannot be replaced.

- Distance between two nodes is computed using Euclidean distance

$$
D=\sqrt{\left(y_{i}-y_{j}\right)^{2}+\left(x_{i}-x_{j}\right)^{2}}
$$

\section{Energy dissipation model}

Energy consumption model utilized in this model is represented in figure, proposed scheme adopts free space $\left(d^{2}\right)$ and multipath $\left(d^{4}\right)$ radio channel energy consumption depending on transmission range between transmitter and receiver [25]. To transfer data packet of $k$ bits at distance $d$, the energy consumption is computed as:

$E_{t_{x}}= \begin{cases}k * E_{\text {elec }}+k * \epsilon_{f s} * d^{2} & d \leq d_{0 y} \\ k * E_{\text {elec }}+k * \epsilon_{a m p} * d^{4} & d>d_{0 y}\end{cases}$

$d_{0}$ is calculated by $d_{0}=\sqrt{\epsilon_{f s} / \epsilon_{a m p}}, E_{a l e c}$ is electronic energy consumed during coding, decoding and modulation of signal. Amplification factor of transmitter circuit is represented as $\epsilon_{f s}$ and $\epsilon_{a m p}$ if $d \leq d_{0}$ and $d>d_{0}$, where $d_{0}$ is threshold. To receive the transmitted data packet of $k$ bits at receiver, the energy consumption at receiver is computed as:

$E_{r_{x}}=k * E_{\text {elec }}$

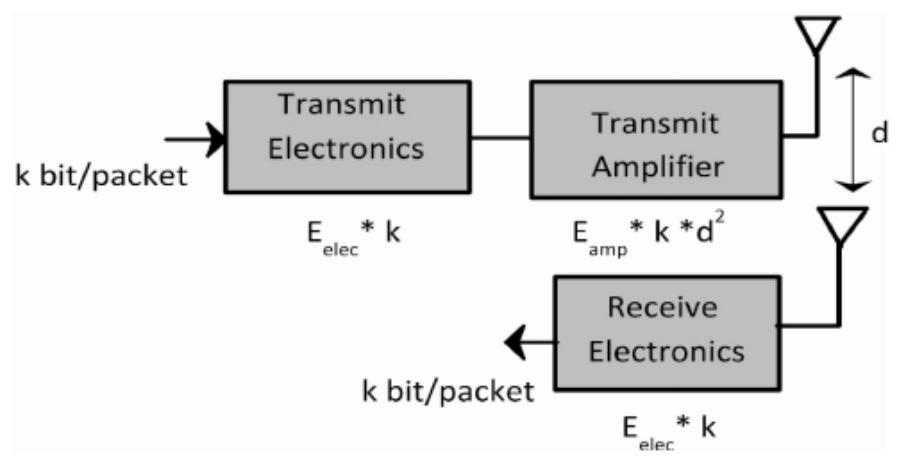

Figure 2: Energy Dissipation Model

\section{Proposed IEECT}

LEACH protocol aims to improve network lifetime by adopting clustering and periodic data gathering which reduces the transmission overhead between nodes and sink. Data aggregation at the cluster head $(\mathrm{CH})$ reduces correlated data locally and optimizes data which result in low energy consumption at node. Time division multiple access (TDMA) scheduling used by LEACH allows cluster members (CM) to save energy by making nodes to go into sleep mode and extends network lifetime. Election of cluster head is determined by computing threshold $t_{h}(n)$ value and random number generation function. Each sensor node generates a random number $q(0 \leq q<1)$. Node selected as $\mathrm{CH}$ if it satisfies $q \leq t_{h}(n)$, where $t_{h}(n)$ is calculates as :

$t_{h}\left(n_{i}\right)=\left\{\begin{array}{lr}\frac{p_{b}}{1-p_{b} *\left(r_{c} * \bmod \left(1 / p_{b}\right)\right)}, & n_{i} \in V_{n} \\ 0, & \text { otherwise }\end{array}\right.$ 
$p_{b}$ represents the probability of each sensor node becoming $\mathrm{CH}$ during $0^{\text {th }}$ round, $r_{c}$ represents current number of rounds, $V_{n}$ is the set of nodes not elected as $\mathrm{CH}$ in recent round of $1 / p_{b}$. The amplified power energy consumption to total energy consumption is represented as:

$$
E_{\text {ratio }}=\left\{\begin{array}{l}
\frac{k * \epsilon_{f s} * d^{2}}{k * E_{\text {elec }}+k * \epsilon_{f s} * d^{2}}, d \leq d_{0} \\
\frac{k * \epsilon_{\text {amp }} * d^{4}}{k * E_{\text {elec }}+k * \epsilon_{\text {amp }} * d^{4}}, d>d_{0}
\end{array}\right.
$$

However, while selecting $\mathrm{CH}$ traditional LEACH does not consider density of nodes, residual energy and average energy, therefore $\mathrm{LEACH}$ fails to provide optimal $\mathrm{CH}$ selection. $\mathrm{CH}$ is directly communicated to sink by adopting single hop communication which consumes more energy and leads to quick exhaustion of node energy. For optimal cluster head formation and to enhance the network lifetime an improved LEACH model is presented.

\section{Improved LEACH}

When the nodes are closer to sink, the energy dissipation model of improved LEACH for $\mathrm{CH}$ per round using free space model is given as :
$R$ is area of distributed nodes, sensor nodes $(\mathrm{SN})$ or cluster member (CM) energy consumption for per round is given as :

$E_{S N}=k * E_{\text {elec }}+k * \epsilon_{f s} * d_{C H}^{2}$

$d_{C H}^{2}$ is expected square distance between sensor node to $\mathrm{CH}$, which is computed as :

$$
\begin{aligned}
& E_{d}\left(d_{C H}^{2}\right)=\iint\left(m^{2}+n^{2}\right) \varphi(x, y) d x d y \\
& =\frac{L^{2}}{2 \pi j}
\end{aligned}
$$

Energy consumption of each cluster is given as:

$$
\begin{aligned}
& E_{g_{\text {cluster }}}=C H_{E_{g}}+\left(\frac{N}{j}-1\right) E_{S N} \\
& \approx C H_{E_{g}}+\frac{N}{j} E_{S N}
\end{aligned}
$$

proposed modified energy consumption during each round is expressed as:

$E_{r}=j E_{g_{\text {cluster }}}$
$C H_{E_{g}}=\left(\frac{N}{j}-1\right) * k * E_{\text {elec }}+\frac{N}{j} * k * E_{C H}+k * E_{\text {elec }}+k\left(2 N E_{\text {elec }}+\frac{1}{2}+N E_{S_{\mathrm{n}}}^{2}+j \epsilon_{f s} d_{s_{\mathrm{n}}}^{2}+N \epsilon_{f s} d_{C H}^{2}\right)$

clusters per round is represented as $j, \frac{N}{j}$ is average node of each cluster, $\mathrm{CH}$ energy consumption on receiving message of 1 bit is given as $E_{\mathrm{CH}}$. Square distance between $\mathrm{CH}$ to sink is denoted as $d_{S_{n}}^{2}$. Assuming that sink node is placed at location $(m, n)$, without loss of generality the node $\varphi(x, y)$ distributed in an area. The square distance between cluster head to sink is calculated as:

$$
\begin{aligned}
& E_{d}\left(d_{S_{n}}^{2}\right)=\iint\left((m-x)^{2}+(n-y)^{2}\right) \varphi(x, y) d x d y \\
& =\iint \frac{(m-x)^{2}+(n-y)^{2}}{R} d x d y
\end{aligned}
$$

Optimal number of cluster head for each round $E_{r}$ with respect to $f$ is given as:

$$
j_{o p t}=\frac{\sqrt{N}}{\sqrt{2 \pi}} \frac{L}{d_{S_{n}}^{2}}
$$

Energy dissipation of $\mathrm{CH}$ for multipath model $d^{4}$ when sink is far from nodes is given as :

$$
C H_{E_{g}}=\left(\frac{N}{j}-1\right) * k * E_{\text {elec }}+\frac{N}{j} * k * E_{C H}+k * E_{\text {elec }}+
$$

Expected distance is computed as: 
$E_{d}\left(d_{s_{n}}^{4}\right)=\iint\left((m-x)^{2}+(n-y)^{2}\right)^{2} \varphi(x, y) d x d y \begin{aligned} & \text { By considering four parameters proposed modified } \\ & \text { LEACH can balance the nodes energy consumption and } \\ & \text { extends network lifetime. }\end{aligned}$ $=\iint \frac{\left((m-x)^{2}+(n-y)^{2}\right)^{2}}{R} d x d y$

proposed modified energy consumption during each round is expressed as:

$E_{r}=j E_{g_{\text {cluster }}}$

$=k\left(2 N E_{\text {elec }}+N E_{C H}+j \epsilon_{a m p} d_{S_{n}}^{4}+N \epsilon_{f s} d_{C H}^{2}\right)$

Optimal number of cluster head for each round $E_{r}$ with respect to $j$ is given as:

$j_{\text {opt }}=\frac{\sqrt{N}}{\sqrt{2 \pi}} \sqrt{\frac{\epsilon_{f s}}{\epsilon_{a m p}}} \frac{L}{d_{S_{n}}^{2}}$

\section{Proposed algorithm to select cluster head $(\mathrm{CH})$}

The selection of $\mathrm{CH}$ in conventional LEACH is done randomly, node with less energy chosen as $\mathrm{CH}$ dies quickly. To avoid early death of nodes and to balance energy consumption the energy threshold is given as:

$$
t_{h}\left(n_{i}\right)=\left\{\begin{array}{lr}
\frac{t_{e}}{1-t_{e} *\left(r_{c} * \bmod \left(1 / t_{e}\right)\right)}, & n \in V_{n} \\
0, & \text { otherwise }
\end{array}\right.
$$

The energy adjustment parameter $t_{B}$ can be expressed as;

$t_{e}=\frac{p_{b} * n_{i} * E_{\text {res }}^{n_{i}} * E_{\text {initial }}^{n_{i}}}{E_{\text {total }}^{n_{\tilde{i}}} * E_{\text {avg }}^{n_{i}}}$

$p_{b}$ is probability of selecting optimal $\mathrm{CH}$, residual energy of current node is represented as $E_{r e s}^{n_{\bar{i}}}$. Initial energy of node is given as $E_{\text {initial }}^{n_{\tilde{i}}}$, total energy of network is represented as $E_{\text {total }}^{n_{\tilde{\mathrm{i}}}}$ and $E_{\text {avg }}^{n_{\tilde{\mathrm{i}}}}$ is the average of sensor nodes, it is obtained by:

$E_{\text {avg }}^{n_{i}}=\frac{E_{\text {total }}^{n_{i}}\left(1-\frac{r_{c}}{r_{c_{\max }}}\right)}{n_{i}}$

\section{Cluster formation algorithm}

After $\mathrm{CH}$ selection, $\mathrm{CH}$ broadcast its join request message to other nodes that they have become $\mathrm{CH}$ and sensor nodes within transmission range can join and form a cluster. Each sensor node decides whether to participate in cluster formation based on the received signal strength of $\mathrm{CH}$. Nodes nearer to sink does not participate in cluster formation and transmit data directly to sink. Sensor node or cluster member (CM) finds the nearest $\mathrm{CH}$ and calculates the distance $d_{C M \text { to } C H}$ between them. If $d_{C M \text { to } C H}>d_{C M \text { to sink }}$ sensor node does not join to any cluster and directly communicates with sink during data transmission. When $d_{C M \text { to } C H}<d_{C M \text { to sink }}$ sensor node joins cluster and acts as cluster member (CM).

\section{ESI-LEACH Algorithm}

1. $n_{i^{-}}$number of sensor nodes, $r_{c}$ - number of rounds

2. Initialize WSN by deploying nodes, assigning energy, MAC parameters

3. compute distance $d_{S_{n}}$

4. for $i=1$ to $r_{c}$

5. $E_{\text {avg }}^{n_{i}}=\frac{E_{\text {total }}^{n_{i}}\left(1-\frac{r_{c}}{r_{C_{\max }}}\right)}{n_{i}}$

6. $t_{e}=\frac{p_{b} * n_{i} * E_{r e s}^{n_{i}} * E_{\text {initial }}^{n_{i}}}{E_{\text {total }}^{n_{i}} * E_{\text {avg }}^{n_{i}}}$

7. $t_{h}\left(n_{i}\right)=\left(1-t_{e} *\left(r_{c} * \bmod \left(1 / t_{\theta}\right)\right)\right.$

8. $R=$ random number

9. if $R \leq\left(t_{h}\left(n_{i}\right)\right)$

10. $n_{i} \rightarrow \mathrm{CH}$

11. Evaluate distance to $\mathrm{CH} d_{C H}$

12. end if

13. if $d_{C H}<d_{S_{n}}$

14. Select $\mathrm{CH}$

15. Initiate data transfer to sink

16. $i=i+1$

17. go to step 4

18. end 


\section{Tree construction and data aggregation}

After cluster head and cluster formation, sink node initiates logical tree construction for optimal routing of aggregated data to sink. Based on the $\mathrm{CH}$ location and energy level the tree is constructed. Sink broadcasts tree formation control message which includes five fields $\left(I D_{S_{n}},-, \infty\right.$, Statu $\left._{S_{n}}, L_{0}\right)$ representing sink id, parent, residual energy, leaf or relay node and level. With sink as the root the aggregation tree of $\mathrm{CH}$ nodes will be formed. Periodically the sink can re-construct tree depending on $\mathrm{CH}$ rotation. TDMA scheduling is used by $\mathrm{CH}$ to transfer data at given slots, if $\mathrm{CH}$ does not have any data to be sent their radios are kept off to save power consumption. The data transmission process starts after tree is formed among CH's. Sensor node or cluster member (CM) sends the sensed data to their respective $\mathrm{CH}$ in time slot, $\mathrm{CH}$ then aggregates the received data from $\mathrm{CM}$ and forwards to next level parent node until data is reached to sink.

\section{Tree formation and data aggregation algorithm}

1. Sink initiates tree formation by broadcasting control message assuming infinite energy

2. $\left(\right.$ ID $_{S_{n^{2}},},{ }_{,}$, Status $\left._{S_{n},} L_{0}\right)$

3. Gathering $\mathrm{CH}$ 's information

4. (CH $-I D$, parent, power, status, level)

5. TDMA scheduling for each $\mathrm{CH}$

6. if no data transmission then

7. turn off radio's

8. else

9. forward aggregated data to next level node rooted to sink

10. Sensor nodes or cluster members $(\mathrm{CM})$ send data to respective $\mathrm{CH}$ using TDMA time frame

11. send ( $C M-I D$, data, data_size, $C H)$

12. if ( $C M$ has no data to send) then

13. initiate sleep mode

14. end if

15. if $(C M-I D \notin$ current $C H)$ then

16. $\mathrm{CH}$ node goes to sleep mode

17. end if

18. send aggregated data to next higher level until it reaches to sink.

\section{Performance Evaluation}

Simulation environment and parameters

Simulation of proposed IEECT is carried out using discrete network simulator tool NS2 [26]. The performance of IEECT is compared with SILEACH [27] and SEECP [28] and performance metrics like packet delivery ratio at sink, delay, energy consumption, network lifetime and overhead are analysed. Table 1 show the detailed parameters used in simulation.

Table 1: Simulation parameters

\begin{tabular}{|c|c|}
\hline Parameters & Value \\
\hline Nodes & 100 \\
\hline Queue type and size & Droptail,50 \\
\hline MAC protocol & 802.11 collision free \\
\hline Simulation Time & $100 \mathrm{sec}$ \\
\hline Deployment Area & $1000 \times 1000 \mathrm{mts}$ \\
\hline Packet Size & 512 bytes \\
\hline Communication Range & 250 mts \\
\hline Protocols & SILEACH and SEECP \\
\hline Traffic Type & CBR \\
\hline Initial Energy & 100 Joules \\
\hline Sink node & 1 \\
\hline Scheduling & TDMA \\
\hline Rounds & 50 \\
\hline
\end{tabular}

\section{Performance metrics}

- Percentage of packets received at sink over number of rounds
Figure 3 illustrates the PDR (packet delivery ratio) at sink node over time. $\mathrm{X}$-axis represents number of rounds and $\mathrm{Y}$-axis represents percentage of packet delivered. With increase of time, it is observed that ratio of received packets at sink is higher in IEECT 
compared to SILEACH and SEECP. This is because formation of tree among $\mathrm{CH}$ to sink, such that the aggregated data can be efficiently delivered to sink. IEECT selects minimum hops to reach sink through connected CH's as sink knows the network topology of connected $\mathrm{CH}$ 's and efficiently utilises bandwidth for data transmission using TDMA and achieves $98 \%$ in delivering packets at sink. Whereas SILEACH has $92 \%$ and SEECP has $89 \%$ of packet delivery percentage at sink Routes to sink in SILEACH and SEECP are discovered using route request messages every time when $\mathrm{CH}$ has data to be sent to sink which has higher communication overhead.

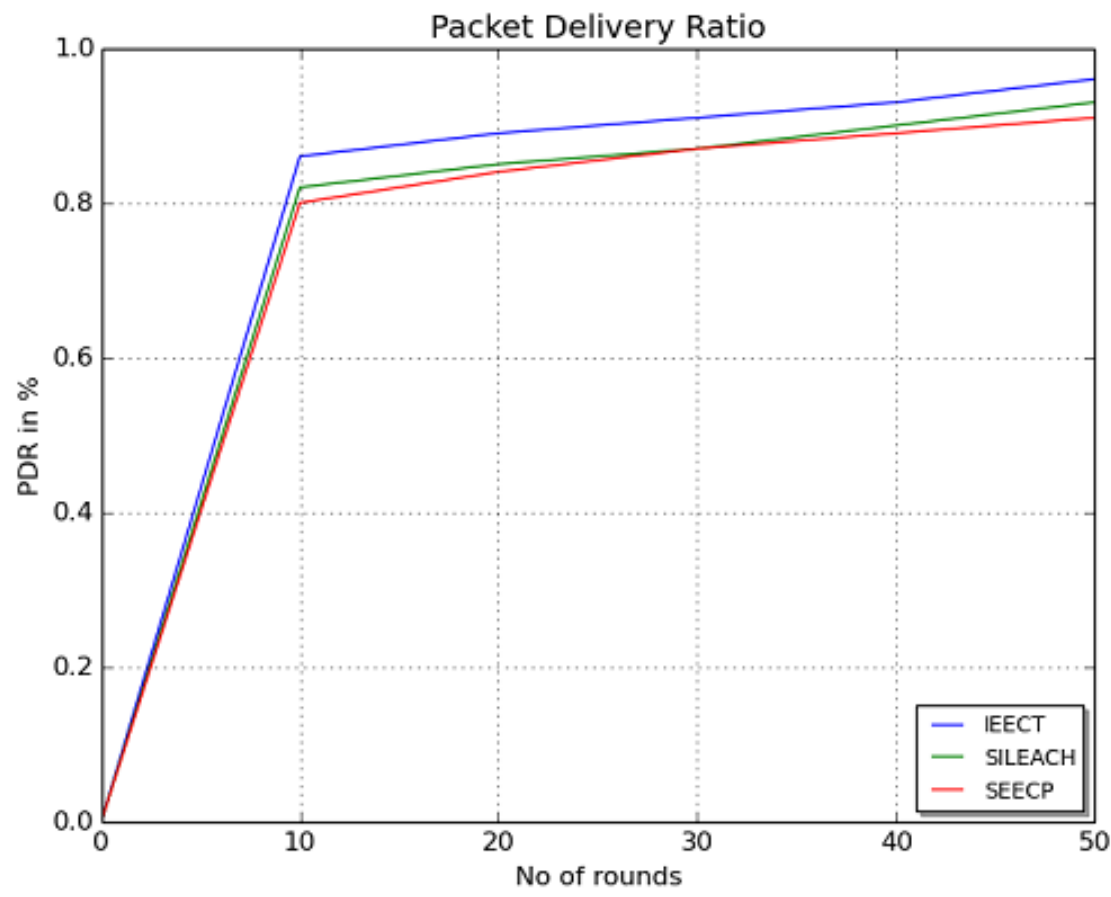

Figure 3: Comparison of packet delivery ratio at sink

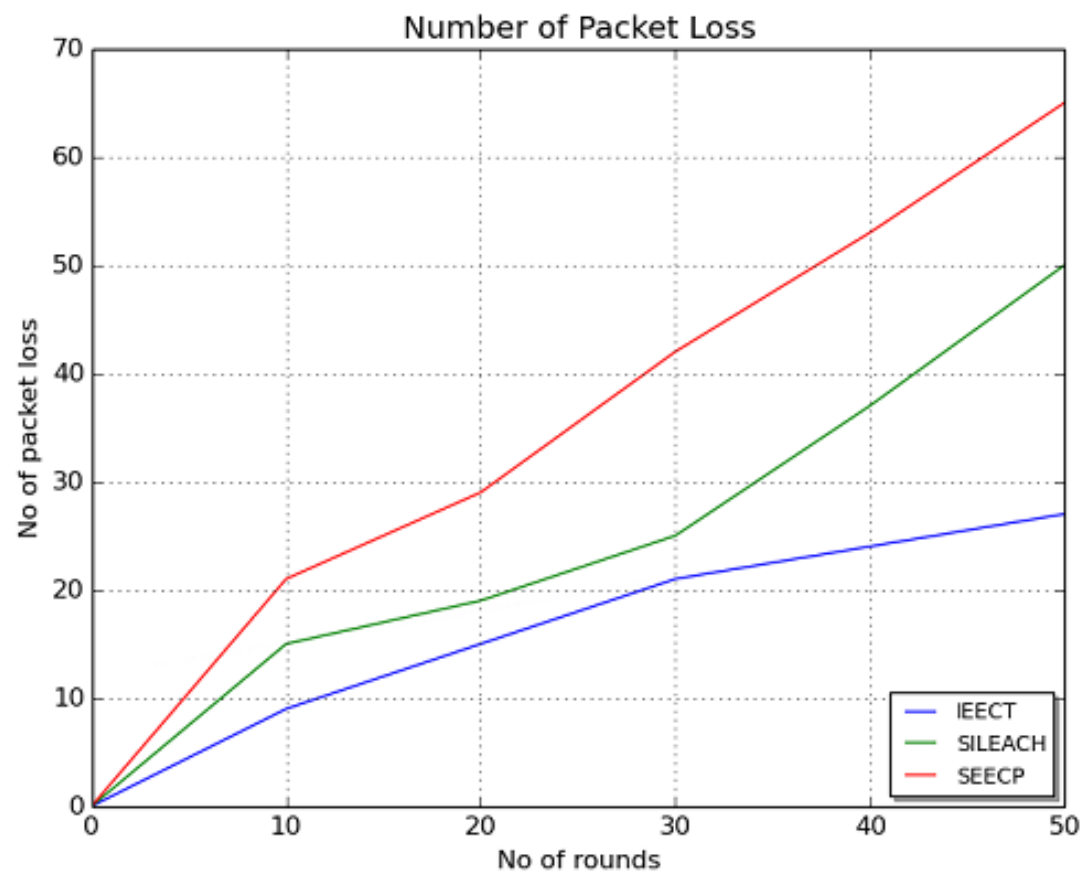


Figure 4: Comparison of packet loss

Figure 4 illustrates the total number of packet loss during simulation rounds. It is observed that the IEECT has lower packet loss compared to SILEACH and SEECP, this is due to less number of channel fading. Proposed IEECT considers free space and multipath loss model such that packet loss is minimized.

\section{- Network lifetime and stability over number of rounds}

Network lifetime is defined as the duration of sensor operation in network from beginning to the death of last sensor node in the network. Nodes energy is consumed during data transmission, which is highly reliant on the routing efficiency used to route data. When a node's energy is fully depleted, it is considered to be dead. Figure 5 illustrates the network lifetime of IEECT compared with SILEACH and SEEP. X-axis indicates the number of rounds and $\mathrm{Y}$-axis indicates the number of dead nodes. It is observed that nodes start to die as number of rounds is increased. It is observed that at $10^{\text {th }}$ round SILEACH has less nodes death compared to IEECT and SEEP initially, while at round $20^{\text {th }}$ SILEACH and SEEP has more dead nodes compared to IEECT. Since the energy balance among node is done properly in IEECT by considering average energy, total network energy, residual energy and initial energy. Considering these parameters avoids early death of node and increases network stability and lifetime of the network. Formation of optimal clusters and tree based routing helps to find optimal route with minimum hop which reduces the number of transmissions and achieve higher stability.

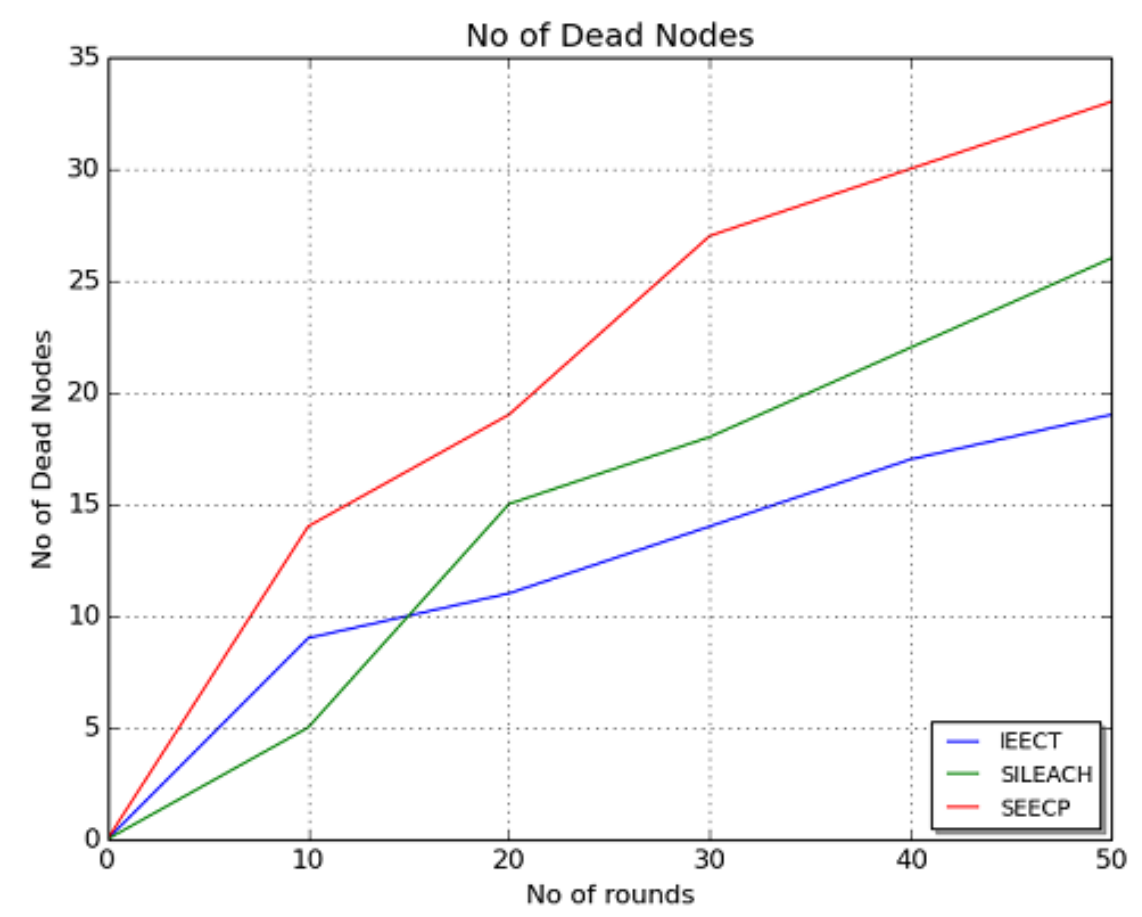

Figure 5: Comparison of dead nodes 


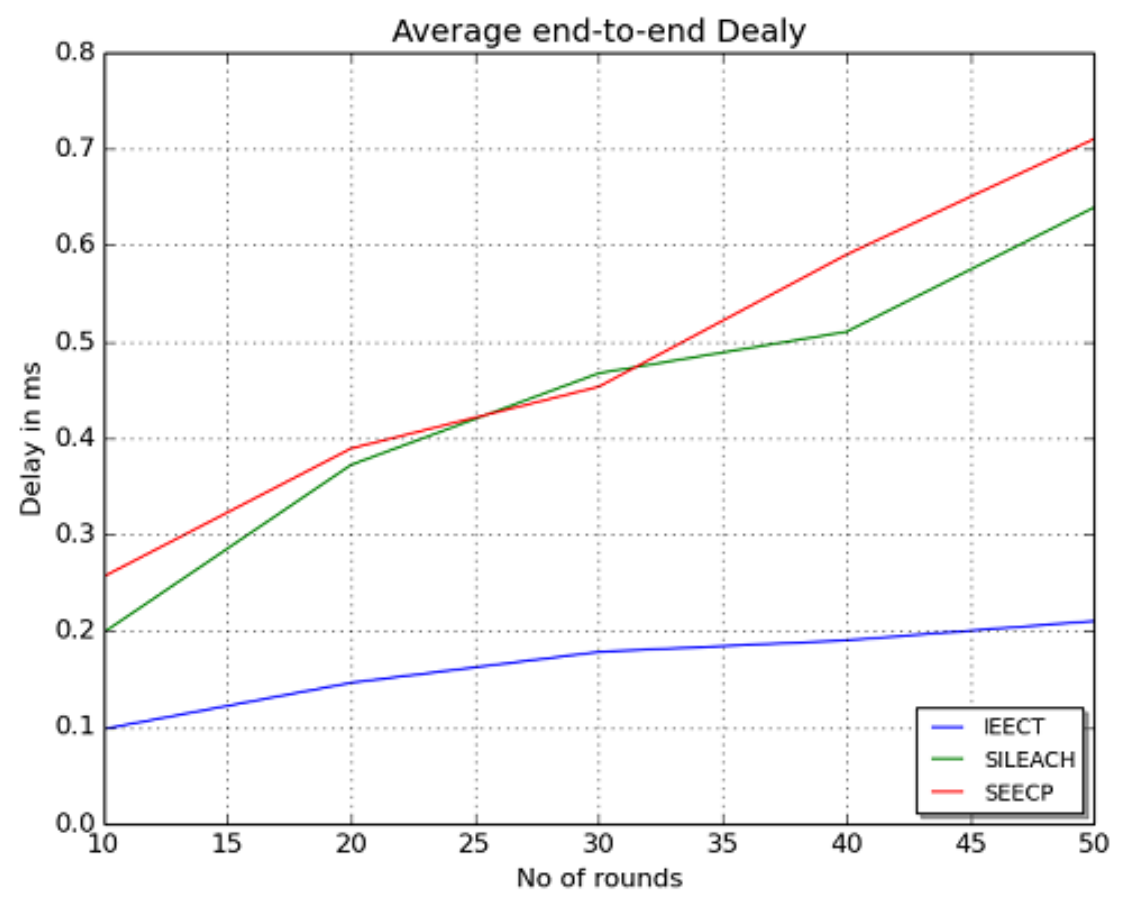

Figure 6: Comparison of average end-to-end delay

\section{- Delay over number of rounds}

Figure 6 illustrates the average delay of IEECT compared with SILEACH and SEEP. X-axis indicates number of rounds and $\mathrm{Y}$-axis indicates average delay in milliseconds. It can be clearly observed the change of delay as number of rounds is increased. Delay fluctuation is more in SILEACH and SEEP due to probabilistic $\mathrm{CH}$ selection and discovering routes to sink for data transmission. However IEECT has significant amount of delay due to optimal $\mathrm{CH}$ selection and tree construction for data transmission to sink among CH. IEECT reduces redundant data transmission during data aggregation. Tree based routing to sink helps to achieve higher data delivery through minimum hops which reduces the overhead of finding routes to sink.

\section{- Energy consumption over number of rounds}

Figure 7 illustrate the energy consumption of IEECT compared SILEACH and SEECP. X-axis indicates number of rounds and $\mathrm{Y}$-axis indicates energy consumption in joules. It is observed that SILEACH consume less power compared to SEECP, this is because SILEACH considers cost function for energy threshold calculation and balances the nodes energy to form clusters. To prove performance of proposed IEECT in terms of energy consumption, it is seen that proposed IEECT calculates threshold energy by considering initial energy, average energy, total network energy and residual energy. By integrating tree based routing avoids retransmission of data packets to sink and enhances the network lifetime. 


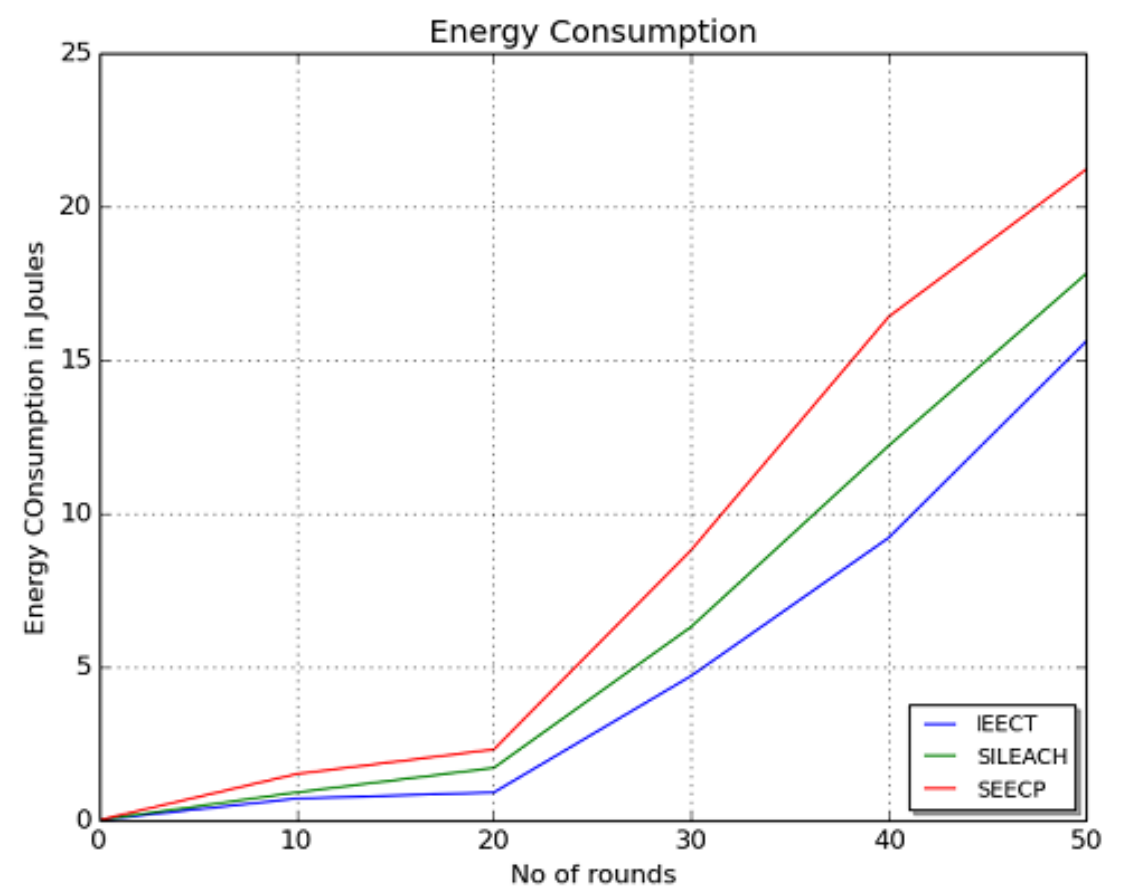

Figure 7: Comparison of energy consumption

- Communication overhead over number of nodes

Figure 8 illustrates the communication overhead of IEECT compared with SILEACH and SEECP. X-axis indicates number of nodes and $\mathrm{Y}$-indicates the overhead. The overhead of IEECT is low compared to SILEACH and SEECP. Communication overhead increases and number of nodes is increased this is due to more route computation process is executed to reach sink. It is observed in the graph IEECT considers the overall network information by forming tree among $\mathrm{CH}$ and optimal cluster formation. IEECT selects optimal routes to reach sink with minimum hop and reduces overall communication overhead.

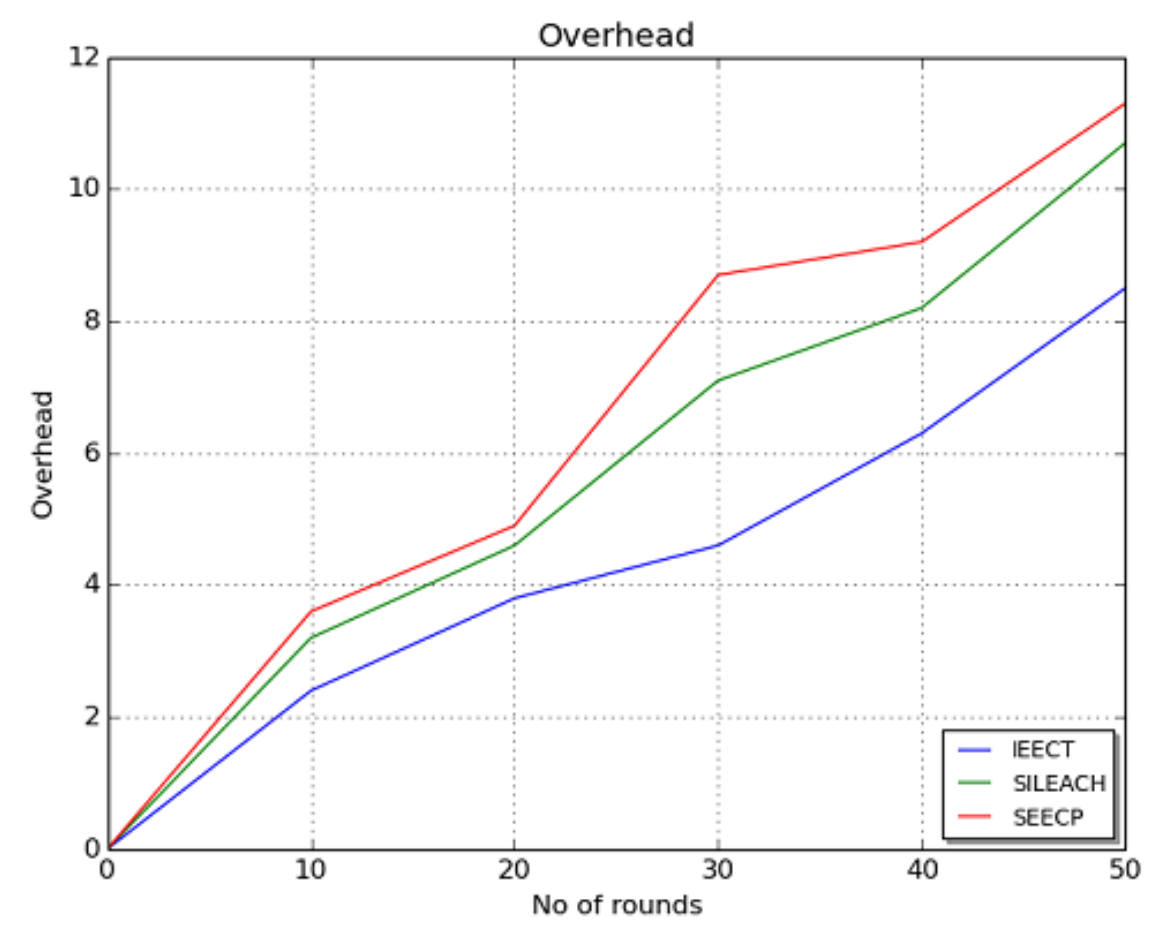




\section{Figure 8: Comparison of communication overhead}

\section{Conclusion}

Due to energy constrain in WSN, extending network lifetime and achieving reliable routing is a challenging issue. Existing clustering methods have been proposed to balance the nodes energy and maximize the network lifetime. However these methods suffer from high communication cost and frequent re-clustering of nodes which leads to energy drain faster. In this paper we propose an improved energy efficient cluster tree (IEECT) based routing by modifying existing LEACH. IEECT considers four parameters such as initial energy, average energy of nodes, residual energy and total network energy to calculate the threshold energy for optimal cluster formation and cluster head selection. Rotation of $\mathrm{CH}$ balances the nodes energy and improves overall network lifetime. Integration of tree based data aggregation routing achieves optimal routing of data to sink. Tree construction among $\mathrm{CH}$ helps to route the aggregated information with minimum hops to sink. The simulation results shows proposed IEECT outperform existing SILEACH and SEECP in terms of energy consumption, packet delivery at sink, delay and communication overhead. In future, solution for fault tolerance of $\mathrm{CH}$ through dynamic adaptation can be attempted to maintain QoS parameters.

\section{References}

[1] Heinzelman, W. R., Chandrakasan, A., \& Balakrishnan, H. (2000). Energy-efficient communication protocol for wireless microsensor networks. Paper presented at the system sciences, proceedings of the 33rd annual Hawaii international.

[2] Yetgin H, Cheung KTK, El-Hajjar M, Hanzo LH (2017) A survey of network lifetime maximization techniques in wireless sensor networks. IEEE Commun Surv Tutor 19(2):828 854

[3] C. Walravens, W. Dehaene, Low-power digital signal processor architecture for wireless sensor nodes, IEEE Trans. Very Large Scale Integration (VLSI) Syst. 22 (2) (2014) 313-321.

[4] T. Wang, M.Z.A. Bhuiyan, G. Wang, M.A. Rahman, J. Wu, J. Cao, Big data reduction for a smart city's critical infrastructural health monitoring, IEEE Commun. Mag. 56 (3) (2018) 128-133.

[5] P.K. Sahoo, S.K. Mohapatra, S.L. Wu, Analyzing healthcare big data with prediction for future health condition, IEEE Access 6 (2016) 9786-9799.
[6] L.D. $\mathrm{Xu}, \mathrm{W} . \mathrm{He}, \mathrm{S}$. Li, Internet of things in industries: a survey, IEEE Trans. Ind. Inf. 10 (4) (2014) 2233-2243.

[7] Z. Shao, J. Cai, Z. Wang, Smart monitoring cameras driven intelligent processing to big surveillance video data, IEEE Trans. Big Data 4 (1) (2018) 105-116.

[8] K. Wang, Y. Wang, X. Hu, Y. Sun, D.J. Deng, A. Vinel, Y. Zhang, Wireless big data computing in smart grid, IEEE Wirel. Commun. 24 (2) (2017) 58-64.

[9] D.E. Bellasi, R. Rovatti, L. Benini, G. Setti, A lowpower architecture for punctured compressed sensing and estimation in wireless sensor-nodes, IEEE Trans. Circ. Syst.

62 (5) (2015) 1296-1305.

[10] W.B. Heinzelman, A.P. Chandrakasan, H. Balakrishnan, An application-specific protocol architecture for wireless microsensor networks, IEEE Trans. Wirel. Commun. 1 (4) (2002) 660-670.

[11] Gheisari, M.; Alzubi, J.; Zhang, X.; Kose, U.; Saucedo, J.A.M. A new algorithm for optimization of quality of service in peer to peer wireless mesh networks. Wirel. Netw. 2019, 6, 1-9.

[12] Nam, D. Comparison Studies of Hierarchical Cluster-Based Routing Protocols in Wireless Sensor Networks. CATA 2020, 69, 334-344.

[13] Aadri, A.; Idrissi, N. An energy e_cient hierarchical routing scheme for wireless sensor networks. Comput. Sci. Inf. Technol. 2017, 137-148.

[14] Zhao, Z.; Xu, K.; Hui, G.; Hu, L. An energyefficient clustering routing protocol for wireless sensor setworks based on AGNES with balanced energy consumption optimization. Sensors 2018, 18, 3938.

[15] Heinzelman, W.R., Chandrakasan, A., \& Balakrishnan, H. (2000). Energy-efficient communication protocol for wireless microsensor networks. In Proceedings of the 33rd annual Hawaii international conference on system sciences (pp. 1-10)

[16] Heinzelman, W.R., Chandrakasan, A., \& Balakrishnan, H. (2000). Energy-efficient communication protocol for wireless microsensor networks. In Proceedings of the 33rd annual Hawaii international conference on system sciences (pp. 1-10)

[17] Kia, G.; Hassanzadeh, A. A multi-threshold long life time protocol with consistent performance for wireless sensor networks. AEU-Int. J. Electron. Commun. 2019, 101, 114127.

[18] Yarinezhad R, Hashemi SN(2019)Arouting algorithm for wireless sensor networks based on clustering and an fpt-approximation algorithm. J Syst Softw 155:145-161 
[19] MazinaniA,Mazinani SM, MirzaieM(2019) FMCR-CT: an energy-efficient fuzzy multi clusterbased routing with a constant threshold in wireless sensor network. Alexandria Eng J 58(1):127-141

[20] Darabkh, K. A., Al-Maaitah, N. J., Jafar, I. F., \& Khalifeh, A. F. (2018). EA-CRP: A novel energy-aware clustering and routing protocol in wireless sensor networks. Computers \& Electrical Engineering, 72, $702-718$.

[21] Mann, P.S.; Singh, S. Optimal Node Clustering and Scheduling in Wireless Sensor Networks. Wirel. Pers. Commun. 2018, 100, 683-708.

[22] Y. Cheng and D. P. Agrawal, "An improved key distribution mechanism for large-scale hierarchical wireless sensor networks," Ad Hoc Networks, vol. 5, no. 1, pp. 35-48, 2007.

[23] Darabkh, K. A., Al-Maaitah, N. J., Jafar, I. F., \& Khalifeh, A. F. (2018). EA-CRP: A novel energy-aware clustering and routing protocol in wireless sensor networks. Computers \& Electrical Engineering, 72, 702-718.

[24] Darabkh, K. A., El-Yabroudi, M. Z., \& El-Mousa, A. H. (2019). BPA-CRP: A balanced power-aware clustering and routing protocol for wireless sensor networks. Ad Hoc Networks, 82, 155-171.

[25] Behera, T.M.; Samal, U.C.; Mohapatra, S.K. Energy-efficient modified LEACH protocol for IoT application. IET Wirel. Sens. Syst. 2018, 8, 223-228.

[26] NS (2009) Network simulator-NS2. http://www.isi.edu/nsnam/ns

[27] S. A. Al-Zubaidi, A. A. Arif_n, and K. A. AlQadhi, "Enhancing the stability of the improved-leach routing protocol for WSNs," J. ICT Res. Appl., vol. 12, no. 1, pp. 1_13, Apr. 2018.

[28] N. Mittal, U. Singh, and B. S. Sohi, “A stable energy efficient clustering protocol for wireless sensor networks," Wireless Netw., vol. 23, pp. 1809_1821, Aug. 2017, doi: 10.1007/s11276-016-1255-6. 